\title{
Understanding the Diversity of Services Based on Users' Identities
}

\author{
Junjun Sun ${ }^{1}$, Feng Liu ${ }^{1}$, He Zhang ${ }^{1}$, Lin $\mathrm{Liu}^{1,}{ }^{1,}$, and Eric $\mathrm{Yu}^{2}$ \\ Key Laboratory for Information System Security, Ministry of Education \\ Tsinghua National Laboratory for Information Science and Technology (TNList) \\ ${ }^{1}$ School of Software, Tsinghua University, Beijing, China \\ linliuatsinghua.edu.cn \\ ${ }^{2}$ Faculty of Information, Toronto University, Toronto, Canada
}

\begin{abstract}
Internet services involve complex networks of relationships among users and providers - human and automated - acting in many different capacities under interconnected and dynamic contexts. Due to the vast amount of information and services available, it is not easy for novice users to identify the right services that fit his purposes and preferences best. At the same time, it is not easy for service providers to build a service with a customizable set of features that satisfies the most people. This paper proposes to further extend the strategic actors modeling framework $i *$ to analyze the diverse needs of users by modeling explicitly the personal characteristics, organizational positions, and service related roles. We assume that service users' needs and preferences are determined by their personal background, organizational roles, and the immediate operational context in combination. In this way, the origin of the diversity of service needs, quality preferences, and usage constraints, can be ascribed and used as a basis to make rationale selection from currently available types of services, and to reconfigure service interfaces and structures. Example usage scenarios ofweb services are used to illustrate the proposed approach.
\end{abstract}

Keywords: Personalization, Context, Service adaptation, i* modeling.

\section{Introduction}

To provide better web services for large scale social events, such as the Olympic Games, or the World Expo, it is important to understand the needs of all involved parties better. User populations of today's web services are becoming more diversified, and it becomes difficult to identify a prototypical user. Diversity in this sense refers to the variety in user needs which involves accommodating users with different skills, knowledge, age, gender, disabilities, disabling conditions (mobility, sunlight, noise), literacy, culture, income, and so on [12]. For example, designingweb site interface needs to consider the users'computing skills and knowledge. Design of search engines can include basic and advanced searches for different users. Web

\footnotetext{
Corresponding Author.
} 
pages can accommodate users from all over the world:e-commerce sitesprovide multilanguage product catalogue, and description tuned to regional requirements. Cognitively impaired users with learning disabilities and poor memory can be accommodated with modest changes in layout and controlled vocabulary. Expert and frequent users could as well benefit fromcustomizations that speeds high-volume users and macros to support repeated operations.

Literally, diversity can be defined as the characteristics that differentiate people as individuals, as well as the characteristics which make them alike. It is considered to indicate variety. In traditional product-oriented design, diversity assumes at least two roles: build assurances of variety and choice into its processes and products, and it can also be the source or catalyst for change. Most of the existing research in service adaptation focuses on the physical aspect of the service's environmentand context, e.g., time, location, as an origin of how users' needs changes. However, in addition to the physical settings, human factors also play an important role in the formation, change and evolution of the users' need.

In existing context-aware computing, context information is often pre-assumed to include a limited number of variables, e.g., time, location, and run-time status of the platform. Although a few context service frameworks [10] also consider the preferences of users, there is a pressing need for frameworks and models to support the analysis and design of complex social relationships and identities. We need to understand where the diversities of users come from, how they determine user needs, how they affect each other under different contexts, and how such information can be used when making service selection, adaptation, and reconfiguration decisions.

In this paper, we propose an approach to further extend the strategic actors modeling language $i *$ to facilitate the analysis of user's identity information and the underlining social context for Internet Services. Using this modeling approach, we are able to represent different types of identities, social dependencies between identity users and owners, service users and providers, and third party mediators. A reasoning process linking the steps of analyzing user's identity, deriving service needs and preferences, drilling down into service constraints, and making service selection and adaptation is introduced. This modeling approach will help service vendors to provide customizable solutions, user organizations to form integrated identity information management solution, system operators and administrators to accommodate changes. Typical scenarios of a map web service for users with diversified needs are used to illustrate the proposed approach.

This paper is structured as follows. In Section 2, we extend the actor concept of the $i *$ modelling framework and introduce an $i *$-Context to explain the social and identity origin of the users' diverse needs in the internet service environment. In Section 3, two scenarios are given to illustrate how the human factors of $i *$-Context will impact the preference-based internet service selection and customization. Section 4 introduces our design for a web service selection and adaptation system. An Olympic map service case study will also be presented to illustrate the reasoning process. Finally, in Section 5 and 6, we review the related work, and conclude the paper with a discussion of limitations and future directions.

\section{Modeling User Diversity in Service Environment}

There are many dimensions in human diversity that beyond obvious differences such as race, gender, age, physical disabilities, and marital status. Less obvious dimensions 
include: education, lifestyle, nationality, religion, political affiliation, culture and skills. To cope with these diversities, we need a comprehensive modeling framework to capture and understand theseuser diversities and to predict users' needs and preferences accordingly.

Strategic actors modeling framework $i^{*}$ could be used to understand the user's preferences towards certain kinds of web-services. However, current $i^{*}$ modeling lacks of an explanation for the origin of the users' diverse needs and preferences. We extend the actor concept in the $i^{*}$ modelling framework to express the user diversity in the IT Service environment. $i^{*}$ will also be introduced to capture modeling these services related user diversity.

\subsection{Actors in Service Environment}

In the strategic actor modeling framework, $i^{*}$, system players are modeled on three different abstraction levels, namely, role, position, and agent [16]. Putting the actor concept into the web service environment, the meaning of three different levels of actors in the $i^{*}$ modeling framework can be further clarified to model various service users on different abstraction levels.

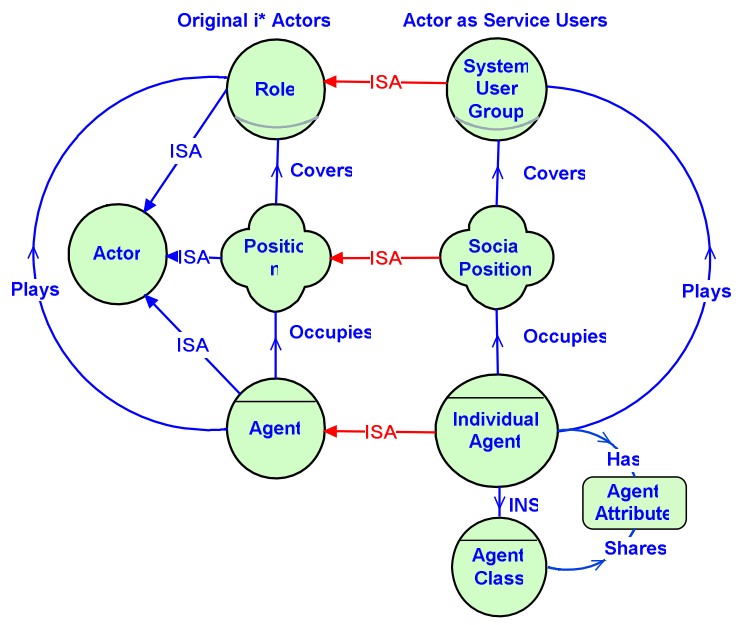

Fig. 1. Mapping service actors

- Service role: In the definition of $i^{*}$ modeling language, a role is defined as "an abstract characterization of the behavior of a social actor within some specialized context or domain of endeavor." [16] Particularly, a role in the web service context could be interpreted as the behavior of a specific group of users towards a certain functionalities of service. For example:

\section{Role[map serviceuser],Role[transportation serviceuser]}

- Social Position: Normally, the term social position is used to represent the social status of an individual. In the service setting, the concept of social position extends 
the positionconcept in $i$ *by referring to aggregations of social roles[7]. It covers not only the organizational perspectives, but also other community-relatedsocial roles and social relations among a group of people. For instance, in the Olympic Games setting, there are:

\section{Position[coach],Position[athlete]}

One could have more than one social position at a time, and social positions all come together with concrete background settings, such as affiliations, certain events or related groups. Different social positions may lead to different user needs and preferences. When there are conflicts between social positions, the actor could compare to find the most important position along with its related events, and select the services accordingly. The importance ofeach particular social positioncould vary as physical context changes.

- Individual Agent: In the original $i^{*}$ modeling framework where agent is defined as "an actor with concrete, physical manifestations, such as human individual." [16].In the service environment, individual agent emphasizes on users, as executers and decision makers of pre-defined services.

\section{Agent $[\mathrm{Jim}]$,Agent[John]}

Agent Attributes: Agent Attributes represent Individual Agent's personal attributes, e.g. age, gender, religion, education level, etc. These agent attributes are comparatively stable and do not change very often. Once the participation of an agent in a social relation relinquishes, the obtained knowledge and history will be transformed into individual attributes.

Attribute[Jim, age $]=20$, Attribute $[\mathrm{John}$, education $]=P h D$

We believeindividual agent's preferences most likely depend on his agent attributes.Although some of these relationships could be clearly defined, most of the agent attribute-preference relationships still remain latent or unknown.

- Agent Class: We use Agent Class to represent an abstract group of people who has the same value for a certain agent attribute which could lead to similar service needs or preferences. Such as:

John INS Agent Class[vegetarian]: Attribute[John, eating habit] = vegetarian

For instance, John is an athlete attending the Paralympics, being a vegetarian, and wants to use the map service to find a nearest restaurant. In this scenario, he is a user of the map service(Role[user [map service]]); his social position is athlete (Position[athlete]); as individual agent, his name is John (Agent[John]), who has a group of concerned attributes: he is a vegetarian (belong to Agent Class[vegetarian]); he is handicapped and uses a wheel chair (belong to Agent Class[wheel chair user]). 


\section{$2.2 i *$-Context for Web Service}

Incorporating results from current context-aware system practices, where location, identity, activity and time related information are used to capture the service context[3]. We propose to extend the current $i^{*}$ concept by putting the position occupation and role playing relation under context. In other words, the agent-position and position-rolerelationship may vary according to physical context such as time, location, etc. Thus, we use service user's social positions and his agent classesas $i^{*}$ Context to capture and model the diversity of service settings.

Table 1. Categories of Social Positions and Agent Classes in $i{ }^{*}$-Context

\begin{tabular}{lll}
\hline & Category & Examples \\
\hline Social Position & Occupation & $\begin{array}{l}\text { Position[athlete], } \\
\text { Position[coach] }\end{array}$ \\
& Family & $\begin{array}{l}\text { Position[father] } \\
\text { Position[daughter] }\end{array}$ \\
& Hobby & Position[golf club member] \\
& & Position[classic music fan] \\
\hline Agent Class & Demographical & Agent Class[male] \\
& Agent Class[teenager] \\
& Cultural & Agent Class[Buddhist $]$ \\
& Physical & Agent Class[wheel chair user] \\
& Education \& Skill & Agent Class[C++ expert] \\
& Habit & Agent Class[vegetarian] \\
\hline
\end{tabular}

Table 1 shows some example categories of social positions and agent classes used in $i$-Context.As individual agent may occupy more than one social positions at a time, the importance of each position under certain physical conditions is also modeled in the $i{ }^{*}$-Context.

Back to our case Study, Table 2 shows the $i *$-Context of a Paralympics athlete, John. Being an athlete and father of a six year old girl, Johnis also avegetarian, Chinese, practicing Buddhism and using a wheel chair.This information brings John the social positions of Position[Father], Position[Athlete], and also reveals the agent classes John belongs to, such as Agent Class[vegetarian], Agent Class[Chinese], Agent Class[Buddhist] and Agent Class[wheel chair user]. Along with these social positions and agent classes, $i{ }^{*}$-Context also provides importance ratings for social positions in various physical contexts. To keep the problem clear, we use a simplified importance value to represent the importance of social positions.

Based on our observation, although individual agents may have more than one social positions at a time, there is usually one position that surpasses all others in importance, and decides what kind of action the agent should performina givenphysical context. Hereby, we introduce the concept of "foreground position".

- Foreground Position: Foreground position is the social position that overweighs all other social positions in importance in a certain physical context for a particular agent.DefinePositions(Agent $[\boldsymbol{a}], \boldsymbol{p c}$ ) asthe set of all social positions Agent $[a]$ plays in certain physical contextpc. 
Foreground Position(Agent $[\boldsymbol{a}], \boldsymbol{p c}): \exists d \in \operatorname{Positions}(\operatorname{Agent}[a], p c) \mid$

$\forall p \in$ Positions(Agent $[a], p c), p \neq d \Rightarrow$ Importance (Agent $[a]$, Position $[p], p c$ ) $<=$ Importance (Agent $[a]$, Position $[d], p c)$

Table 2. Paralympics Athlete John's $i{ }^{*}$-Context

\begin{tabular}{|c|c|c|}
\hline Category & \multicolumn{2}{|l|}{ Value(s) } \\
\hline Social Positions & \multicolumn{2}{|l|}{$\begin{array}{l}\text { Position[Athlete] } \\
\text { Position[Father] }\end{array}$} \\
\hline Agent Class & \multicolumn{2}{|c|}{$\begin{array}{l}\text { Agent Class[vegetarian] } \\
\text { Agent Class[wheel chair user] } \\
\text { Agent Class[Buddhist] } \\
\text { Agent Class[Chinese] }\end{array}$} \\
\hline Position & Importance Factor & Physical Context(Time, Location) \\
\hline Position[Athlete] & 3 (Very Important) $(+)$ & $\begin{array}{l}\text { Time: }\left\{9 \mathrm{am} \sim 11 \mathrm{am}, \operatorname{Aug} 30^{\text {th }}, 2012\right\} \\
\text { \&Location: at Venue }\end{array}$ \\
\hline Position[Athlete] & 2 (Important) & Location: at Gym \\
\hline Position[Athlete] & 0 (Not Important) & Time: $\left\{\right.$ Aug $\left.31^{\text {st }} \sim \operatorname{Sept} 4^{\text {th }}, 2012\right\}$ \\
\hline Position[Father] & 3 (Very Important) $(*)$ & Time: $\left\{\right.$ June $\left.30^{\text {th }}\right\}$ \\
\hline Position[Father] & 2 (Important) & Location: at home \\
\hline Position[Father] & 1 (Less Important) & Time: $\left\{\right.$ Aug $25^{\text {st }} \sim$ Sept $\left.4^{\text {th }}, 2012\right\}$ \\
\hline
\end{tabular}

(+) John's competition is scheduled on the morning of Aug $30^{\text {th }}, 2012$

(*)June $30^{\text {th }}$ is the birthday of John's daughter

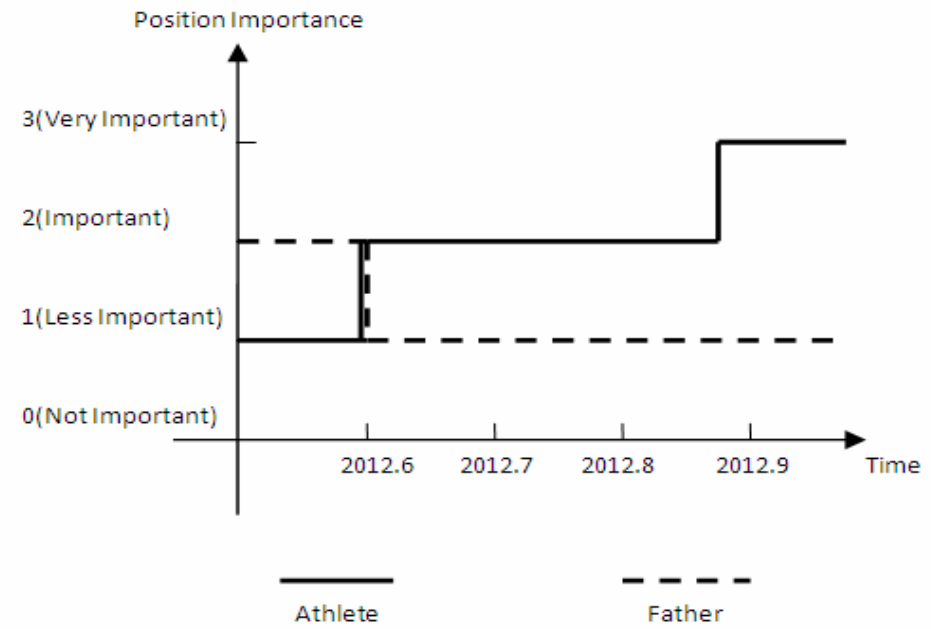

Fig. 2. Foreground Social Positionof John 
For example, as shown in Fig. 2, the importance of John's social position changes according to time.Before 2012.6, John was with his daughter and had not started his intensive Paralympicstraining yet. In this case, during that period of time, Position[father] surpasses Position[athlete] to be the foreground position of John's. However, after 2012.6,John leaves home to take part in a national Paralympics training camp in Beijing, and finally join the wheel fencingcompetition. During these three months, John's social position as Position[athlete] is more important, and hereby it replaces Position[father] to become his foreground position.

\section{Bridging User Diversity and Service Diversity}

In this section, we will explore how the service diversity will comply with the user diversity, and will propose a set of $i$-modeling scenarios to illustrate the propagation of the users' diverse needs under the web service environment. We are in the belief that the users' diverse requirements towards web services originate from the users' social positions and their individual characters defined in its unique $i^{*}$-Context. This diversity will then propagate through the service selection and customization processes, and will eventually lead to the variations in the choice of services and their settings.

\subsection{From User Diversity to Service Diversity}

Fig. 3 shows how the user's own diversity will affect the service selection and customization process. User's needs aremainly decided by the social positions that the user occupies at the time, and it will then become the major concerns for service selection; while the user's preferences are mostly beingaffected by the user's agent attributes, and will further be used to customize the alternative services.

In the following two parts, we will use two particular scenarios to explain how the user's social position will influence the service selection, and how the user's agent attributes will affect the service customization process.

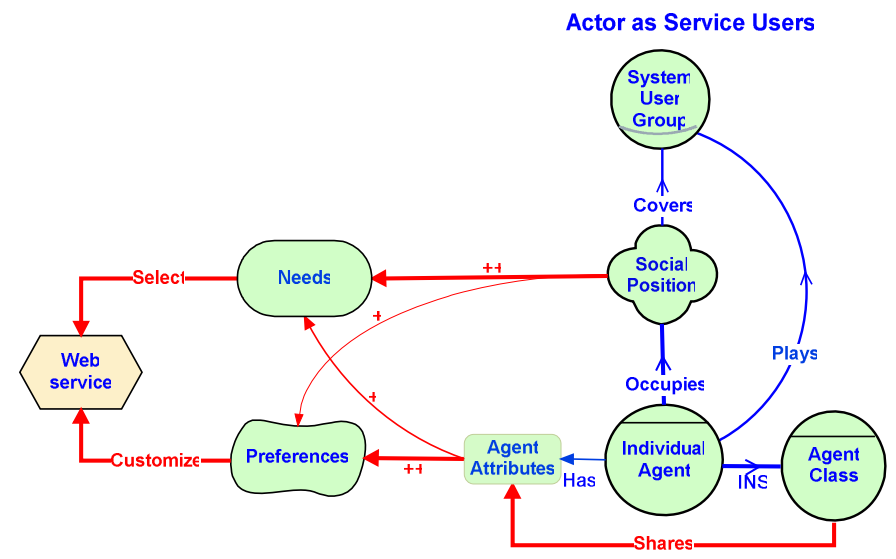

Fig. 3. Propagation Path from User Diversity to Service Diversity 


\subsection{Social Position's Influence over Service Selection}

In this scenario, we will explore how the service user's social position can decide the user's needs and eventually influence over service selection. To make the relationship between social position and services-selections clearer, we choose the same individual agent occupying different positions in the same physical context.

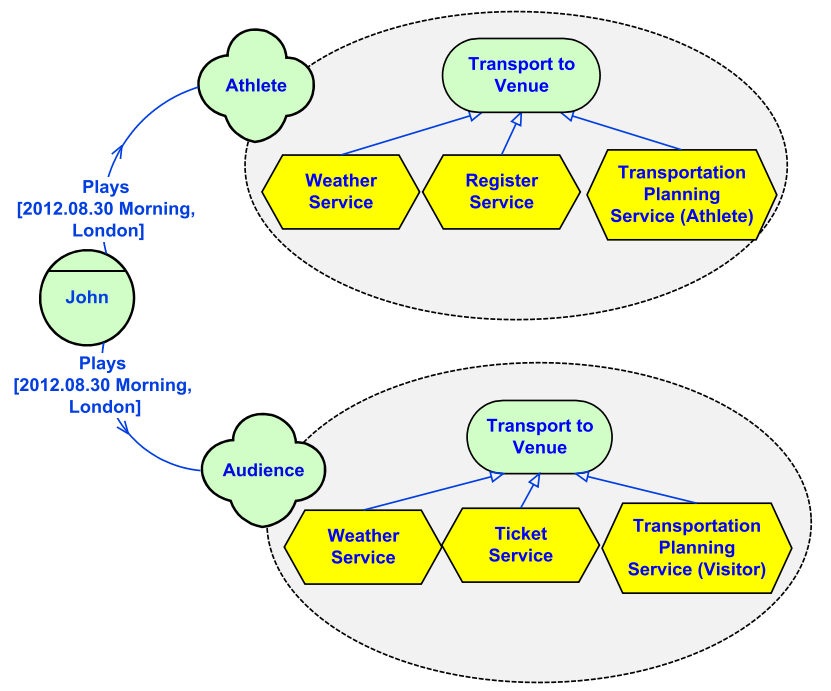

Fig. 4. Social Position's Influence over Service Selection

As depicted in the Fig. 4, during London Olympics, John is aspectator as well as anathlete.Service discovery follows a similar process as the $i^{*}$ goal-task iterative refinementprocess. The top goals in the goal decomposition structure stands for the high level abstract user needs. As these goals are refined into more detailed levels, they will finally reach a level of abstraction that refers to concrete tasks which could matchgiven web services.As shown in Fig. 4, even if the social position spectator and athleteshare the same top goal "Transport to Venue", they could also lead to different service selections. This scenario shows, there are basically three possible results for the same user to pursue the same top -level goal using different positions:

1. The derived services are exactly the same, such as the "weather service", where social positions make no difference.

2. The selected services are completely different, such as the "register service" and "ticket service", which implicates social position leads to completely different procedures for achieving the same goal.

3. The selected services are basically the same type, but have different variations, such as the "transportation planning service" has different entries for athletes or spectators. 
As we can see throughthis analysis, it is important to capture as much information about user's social identity as possible to make rationale service decisions. At the same time, users should be aware of the implications of his social identities when requesting services.

\subsection{Agent Attributes' Influence over Service Customization}

In the second scenario physical context and the social position are the same, but there are two different individual agents. Two individual agents Alan and Steve have the same social position as the Position[athlete]. Their diverse service preferences could be derived from theirindividual attributes respectively.

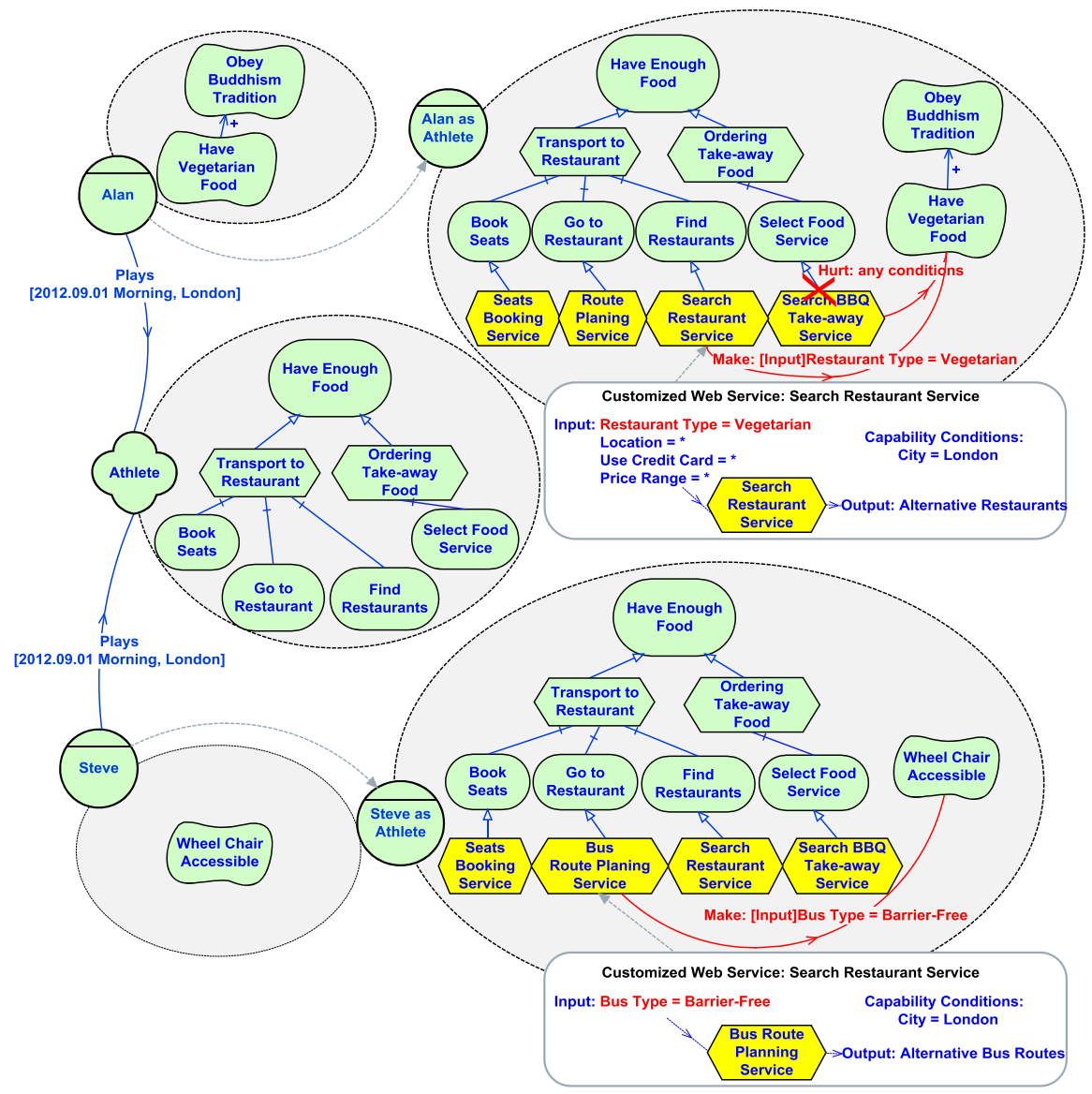

Fig. 5. Individual User Attributes' Influence over Service selection and Customization

In Fig.5, Alan and Steve's agent attributes will propagate to their social position athlete. In $i^{*}$ model, the user preferences deduced from the user's Softgoals could be derived from individual attributes. Now we will use the Softgoal to select and configure web service. 
Fig. 5 shows different individuals with the same social position and the same physical context may lead to different web service choices. In the selection case, take the example of contribution link from Service[Search BBQ Take-away Service] to Softgoal[Vegetarian], a service may not be selected as it hurts the user's softgoal. In the configuration case, such as contribution link between Service[Search Restaurant Service] to Softgoal[Vegetarian], the vegetarian setting should be add to the service input of Service[Search Restaurant Service].

\section{Web Service Selection and Customization using i*-Context}

We have discussed how the user diversity captured by the $i^{*}$-Context would influence the serviceselection and customization. In this section, we propose a systematic process to select and customize services according to the users'personal needs and preferences.

\subsection{Web Service Selection and Customization Process}

Fig.6 shows the main process of our $\mathrm{i}^{*}$-Context based web service selection and customization approach. This process consists of four different steps, namely: Obtaining $i^{*}$-Context; Creating Goal-Task Decomposition Tree; Generating Softgoal Decomposition Structure; and Selecting and Customizing Services. In the following part of this section, we will use a case study to illustrate this process.

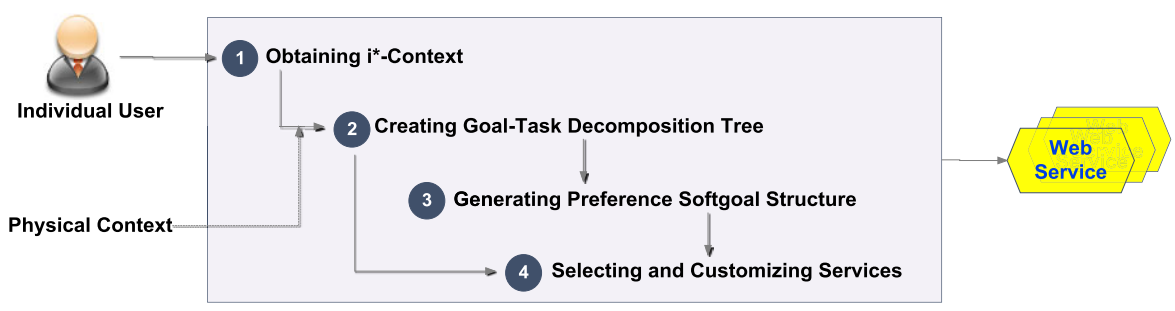

Fig. 6. Service Selection and Customization Process

\subsection{Case Study}

In the previous sections, we have described how the users' diverse needs propagate through the web service selection and customization operations. In this section, we will propose a systematic process to help the service users choose and personalize web services according to their diverse needs. A case study as well as a demo system will be given to illustrate this process.

In our case takesJohn as anexample, according to John's $i^{*}$-Context provided in Table 2:John is a Chinese athlete in the 2012 Paralympics Games; heis also a father to a six year old girl. As an individual agent, Johnis vegetarian, Chinese, using a wheel chair andpracticing Buddhism. 


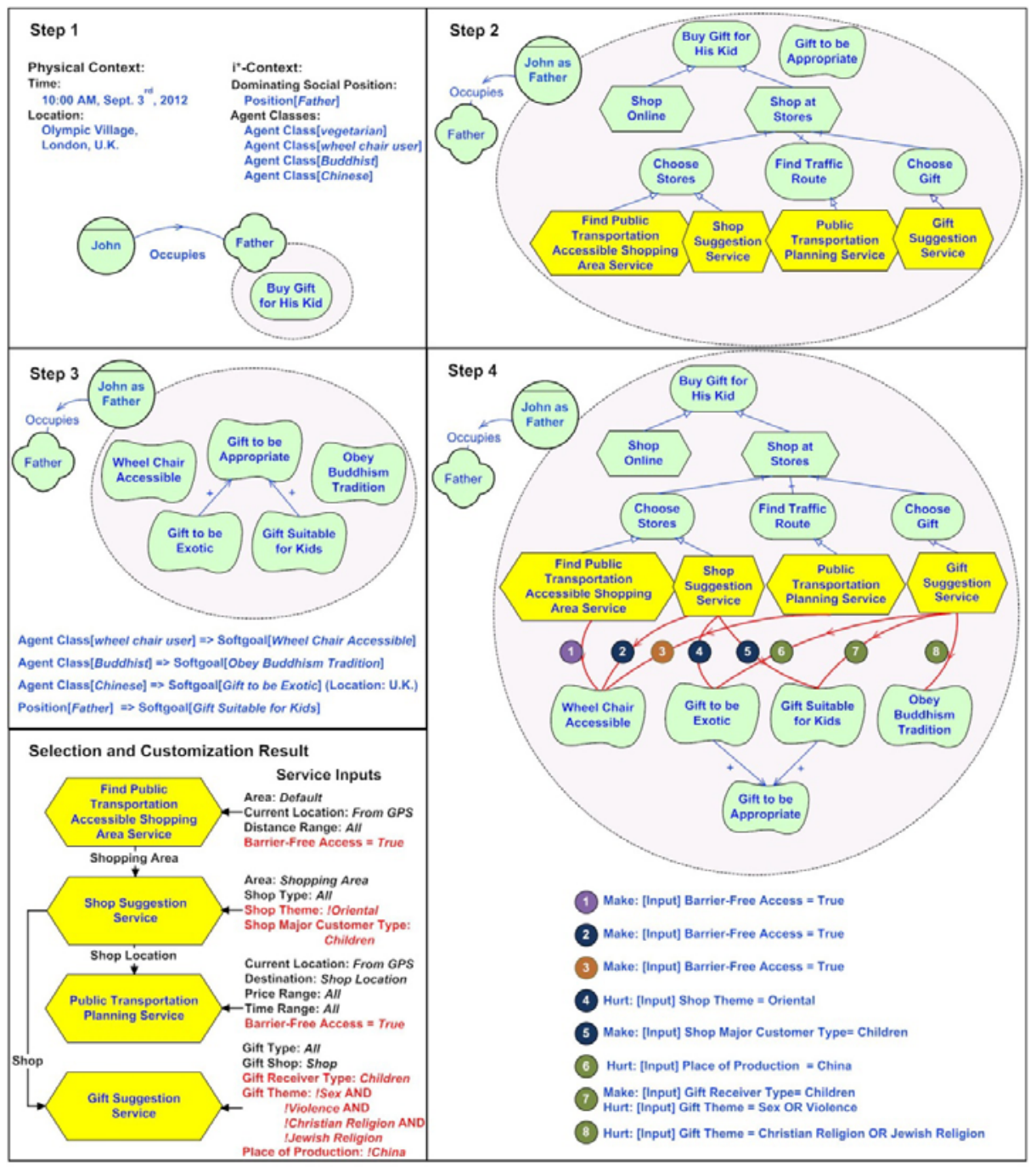

Fig.7. Service Selection and Customization Case Study

As shown in Fig. 7, with the process introduced in the previous part, services selection and customization for our case study will be carried out in the following four steps:

\section{Step 1. Obtaining i*-Context:}

In this step, $\mathrm{i}^{*}$-Context will be obtained from the user's profile. Meanwhile, the user's foreground social position as well as hiscurrent top goal will also be derived or collected. 
In John's case study, when John uses the system, the physical context is 10:00AM, Sept $3^{\text {rd }}, 2012$, in the Olympic Village in London according to the sensors. Based on John's $\mathrm{i}^{*}$-Context (Table 2), his foreground social position is Position[Father]. This is because he has already finished his race, and now doing something to compensatefor being away from his little daughter. According to his to-do list as Position[Father], buying a gift for his daughter is on the top of the list.

\section{Step 2. Creating Goal-Task Decomposition Tree:}

The second step of the process focuses on the user's needs, and uses iterate refinement technique to create a goal-task decomposition tree based on the top user's goal.

In John's case study, Goal[Buy Gift for His Kid] could be satisfied either online or at a gift shop. When we look into Task[Shop at Stores], the task could then be further decomposed into three separate sub-goals, namely, Goal[Choose Stores], Goal[Find Traffic Route] and Goal[Choose Gift]. After that, these sub-goals are being operationalized by a set of different web services (modeled as the bottom-level yellow hexagons). In this step, softgoals derived from goal and task decomposition will also be modeled (see Softgoal[Gift to be Appropriate]).

\section{Step 3. Generating Preference Softgoal Structure:}

In the third step of the process, preference softgoals are being deduced from user's agent classes, his foreground position and the physical context.

As shown in Fig. 7, according to the pre-defined preference deduction rules, John's preference softgoal structure can be generated. For instance, Softgoal[Wheel Chair Accessible] is introduced since hebelongs to Agent Class[wheel chair user]. While as Softgoal[Gift to be Exotic] is derived from the existing Softgoal[Gift to be Appropriate], because John is from China and he is currently in U.K. Two other preference softgoals can be acquired in the same way.

\section{Step 4. Selecting \& Customizing Services:}

In the last step of the process, the alternative services obtained from step 2 will be further selected and customized to better comply with the user's preference softgoals.

As the bottom-level service tasks and the preference softgoal being identified, the relationships between these services and softgoals could be used for service selection and customization. Assuming all the web services are stateless services, the relationships can be represented with the existing conditions of the contribution links in between. Take relationship number 3 as an example, Service[Public Transportation Planning] could make the Softgoal[Wheel Chair Accessible] possible, only if the information service provide a barrier-free access route. Thefinal service selection and customization result after this process is shown in the lower left corner of Fig. 7.

\section{Related Work}

We discuss related work in three areas: agent intentional modelling in requirements engineering, context-aware web services research and preference-based service selection and customization practices. 
In information systems and software engineering research, organizational modeling has been of interest, often in connection with requirements engineering. Goal and agent oriented approaches have been used in this context, and agents or actors are often part of the modeling ontology [13][6]. However, the $i *$-Context approach is distinctive in its treatment of agents/actors as being strategic and context-sensitive[5], and thus readily adaptable to the service requirements adaptation based on identity illustrated in this paper.

Context-aware system and web service are also a very important issue in recent years. Regarding the definition of context for web services, OASIS's WS-Context Standard defines service context as the kind of information that explains "what an activity is and what services it will require in order to perform that work, will depend upon the execution environment and application in which it is used[10].'Dey and Abowd surveyed the existing work in context-aware computing, and have defined context to be any information that can be used to characterize thesituation of an entity. Location, identity, time and activity are the four primary context types that they have summarized.[3]. In Baldauf, Dustdar and Rosenberg's survey, they summarized the different design principles and context models for context-aware systems. According to their survey, the context models used by existing context-aware systems involves key-value models, markup scheme models, graphical models, object oriented models, logic based models and ontology based models [2]. Comparing to the existing context-awareweb service practices covered by these surveys, our approach focuses more on exploring the origin of the users diverse needs and preferences rather than summarizing the directly related context of some specific kinds of services.

There are also a research and practiceson preference-based web service selection and customization. By utilizing decision tree algorithm, Hong, Suh, Kim and Kim have proposed an agent based framework for providing the personalized services using context history [4]. While in Lamparteret. al.'s [14] and Medjahed et. al.'s [8] research, ontology based modelling and reasoning approaches are being introduced to configure the service and system according to user's preferences.

Finally, there is a body of literature on personal and contextual requirements engineering. Sutcliffeet. al. proposed a framework for requirements analysis that accounts for individual and personal goals, and the effect of time and context on personal requirements[1]. The implications of the framework on system architecture are considered as three implementation pathways: functional specifications, development of customizable features and automatic adaptation by the system.Salifuet. al. presents a problem-orientedapproach to represent and reason about contextualvariability and assess its impact on requirements[9]. Ali et. al. proposes a goal-orientedRE modeling and reasoning framework for systems operatingin varying contexts[11]. Liaskoset. al. proposes a variability intensive approach to goal decomposition supporting requirements identification for highly customizable software [15]. Contextualgoal models are introduced to relate goals and contexts;reasoning techniques to derive requirements reflectingthe context and users priorities at runtime; and finally,design time reasoning techniques to derive requirementsfor a system. While these works are all along the same line with our approach, we emphasis on the user's social and personal identities to rationalize the diverse needs they may have when using web services. 


\section{Conclusion and Discussion}

We have outlined an approach for modeling and analyzing diversities of web service users. The approach is based on social and intentional analysiscentered on actor's social and personal identities under different contexts.It allows us to go beyond mechanistic behavior, to deal with the opportunistic and rationaledecision making of strategic actors. Interdependencies among actors' identitybring opportunities as well as place constraints on their service privileges. Strategic actors seek to achieve goals (hard and soft) by obtaining new identities from service providers, taking into account the opportunities and disadvantages arising from various casual relationships, as illustrated in the examples.

Our approach is complementary to existing frameworks and techniques in service personalization and context-aware service provision. Weemphasizethat the systematic analysis of relationships among social rolesand personal background for a given individual actor may play an essential role in eliciting needs for service users. It supports the exploration and management of service alternatives, based on a balanced consideration of all competing requirements, thus complementing the various solutions of recent service selection and adaptation techniques.

While this paper has outlined some basic modeling concepts, much remains to be done.To evaluate the effectiveness of the proposed approach, we are currently developing an application for integrating online banking information services based on user's uniquebackground and settings.

There are also several options to further extend our initial approach. Identity management is increasingly connected with other activities in enterprise management. The proposed diversity rationalization approach provides a way of linking identity related analysis to serviceneeds analysis and technology configuration analysis. The conceptual modeling approach can thus provide a unifying framework for service broker systems, supporting decision making and the management of changes across technical services development, business services model development, and identity management.

Meanwhile, there is also much potential in the synergy between social position modeling and the foundational principles in context modeling. For example, in analyzing the implications of an identity, one would like to model the interrelatedness among their subject matters. The interaction between social concepts and relationships (actors, goals, preferences) and physical ones (e.g., processes, information assets, time, etc.) need to be detailed. Libraries of domain knowledge, service design knowledge with regard to identity management and context management would be very helpful during modeling and analysis. These are topics of ongoing and future research.

\section{References}

1. Sutcliffe, A., Fickas, S., Sohlberg, M.M.: Personal and ContextualRequirements Engineering. Proceedings. In: 13th IEEE International Conference on Requirements Engineering, pp. 19-30 (2005)

2. Baldauf, M., Dustdar, S., Rosenberg, F.: A survey on context-aware systems. International Journal of Ad Hoc and Ubiquitous Computing 2(4), 263-277 (2007) 
3. Gregory, D., Abowd, A.K.: Towards a better understanding of context and contextawareness. In: Gellersen, H.-W. (ed.) HUC 1999. LNCS, vol. 1707, pp. 304-307. Springer, Heidelberg (1999)

4. Hong, J., Suh, E.-H., Kim, J., Kim, S.: Context-aware system for proactive personalized service based on context history. Expert Systems with Applications 36(4), 7448-7457 (2009)

5. Keidl, M., Kemper, A.: Towards context-aware adaptable web services, p. 55. ACM Press, New York (2004)

6. Khalid, H.: Embracing diversity in user needs for affective design. Applied Ergonomics (2006)

7. Masolo, C., Vieu, L., Bottazzi, E.: Social roles and their descriptions. In: Proceedings of Principles of Knowledge Representation and Reasoning (2004)

8. Medjahed, B., Atif, Y.: Context-based matching for Web service composition. Distributed and Parallel Databases 21(1), 5-37 (2006)

9. Salifu, M., Yu, Y., Nuseibeh, B.: Specifying Monitoring and Switching Problems in Context. In: RE 2007, 211-220 (2007)

10. OASIS, Web services context (WS-Context) Standard. OASIS Standards (2003)

11. Ali, R., Dalpiaz, F., Giorgini, P.: A goal-based framework for contextual requirements modeling and analysis. Requir. Eng. 15(4), 439-458 (2010)

12. Schneiderman, B.: Universal usability. Commun. ACM 43(5), 85-91 (2000)

13. Shivakumar, N., Jannink, J., Widom, J.: Per-user profile replication in mobile environments: Algorithms, analysis, and simulation results. Mobile Networks and Applications 2 (1997)

14. Lamparter, S., Ankolekar, A., Studer, R., Grimm, S.: Preference-based selection of highly configurable web services. In: Proceedings of the 16th International Conference on World Wide Web - WWW 2007. ACM Press, New York (2007)

15. Liaskos, S., Lapouchnian, A., Yu, Y., Yu, E., Mylopoulos, J.: On Goal-based Variability Acquisition and Analysis. In: Proceedings of the 14th IEEE International Conference on Requirements Engineering (RE 2006), pp. 76-85 (2006)

16. $\mathrm{Yu}, \mathrm{E}$.: Modelling strategic relationships for process reengineering. $\mathrm{PhD}$ Thesis, University of Toronto, Department of Computer Science (1995) 